\title{
Superconducting thin films of (100) and (111) oriented indium doped topological crystalline insulator SnTe
}

\author{
Weidong Si ${ }^{*}$, Cheng Zhang, Lijun Wu, Genda Gu, and Qiang $\mathrm{Li}^{*}$ \\ Condensed Matter Physics and Materials Science Department, \\ Brookhaven National Laboratory, Upton, New York 11973, USA
}

Recent discovery of the topological crystalline insulator SnTe has triggered a search for topological superconductors, which have potential application to topological quantum computing. The present work reports on the superconducting properties of indium doped SnTe thin films. The (100) and (111) oriented thin films were epitaxially grown by pulsed-laser deposition on (100) and (111) $\mathrm{BaF}_{2}$ crystalline substrates respectively. The onset superconducting transition temperatures are about 3.8 $\mathrm{K}$ for (100) and 3.6 K for (111) orientations, slightly lower than that of the bulk. Magneto-resistive measurements indicate that these thin films may have upper critical fields higher than that of the bulk. With large surface-to-bulk ratio, superconducting indium doped SnTe thin films provide a rich platform for the study of topological superconductivity and potential device applications based on topological superconductors.

*Corresponding authors’email: wds@bnl.gov (WS) and qiangli@bnl.gov (QL). 
Energy band inversion and time-reversal symmetry are the major characteristics to the traditional topological insulators (TIs) [1-5]. The discovery of TIs immediately leads to the search for topological superconductors, which have a full pairing gap in the bulk and gapless surface states [6-9]. Recently a new class of TIs was predicted, topological crystalline insulators (TCI), whereby a band crossing is protected by the crystal point-group symmetry instead of time-reversal symmetry $[10,11]$. Angle-resolved photoemission studies quickly confirmed that the SnTe and (PbSn)Te are TCIs, with the band crossings protected by the crystalline mirror symmetry [12]. Since the time-reversal breaking topological superconductors have attracted great interest due to their relationship with non-Abelian statistics and potential application to topological quantum computation [5], this, in turn, has triggered the search for a topological superconductor in the SnTe system [13].

The indium doped SnTe or (PbSn)Te are superconductors with the superconducting transition temperature, $T_{\mathrm{c}}$, as high as $\sim 4.7 \mathrm{~K}$, depending on the indium doping level [14-19]. It was generally believed that low indium doping may present an unconventional superconductor while high doping leads to a more conventional superconductor [19]. However, due to the small surface-to-bulk ratio, it is difficult to conclude whether or not the topological surface state exists in these materials. Thus low dimensional samples such as thin films or nano-ribbons, which have a much larger surface-to-bulk ratio, were proposed for the measurements [20]. Successful growth of thin films is also vital for future devices based on TIs or TCIs [21, 22]. For integrated device applications like quantum computing thin films are most desirable. Another interesting thin film application would be as the interface between a TI and a conventional superconductor, to induce the superconductivity in the surface of the TI by the proximity effect, another way of producing topological superconductivity [5]. 
Unlike the traditional topological insulators $\mathrm{Bi}_{2} \mathrm{Te}_{3}$ and $\mathrm{Bi}_{2} \mathrm{Se}_{3}$, which possess a single surface state, TCIs possess multiple surface states [10]. For SnTe, four surface states are present on each of (100), (111) and (110) surfaces. There are a number of studies on the fabrication of epitaxial thin films by molecular beam epitaxy of SnTe [23-25]. Surface states have been confirmed in these thin films by magneto-transport measurements, such as Shubnikov-de Haas oscillations and anti-localization effects. Taskin et al. have made (111) oriented epitaxial SnTe thin films on $\mathrm{Bi}_{2} \mathrm{Te}_{3}$ buffer layers on sapphire substrates and observed two dimensional Shubnikov-de Haas oscillations, which suggest a high mobility surface channel [24]. Assaf et al. reported (100) oriented epitaxial SnTe thin films grown on (100) $\mathrm{BaF}_{2}$ substrates. They found anti-localization effects, indicating a mixture of surface states and bulk states [25]. However, there is currently no report on the growth of superconducting indium-doped SnTe thin films.

In this Letter, we report on the magneto-transport properties in superconducting $\mathrm{Sn}_{0.55} \mathrm{In}_{0.45} \mathrm{Te}$ (SIT) thin films successfully grown on (100) and (111) $\mathrm{BaF}_{2}$ substrates. This high doping level has the highest $T_{\mathrm{c}}$ among the indium doped SnTe. High indium doping is generally believed to be a conventional superconductor [19], based on the bulk sample measurements, where surface to bulk ratio is very small. Our motivation of choosing the high doping level in thin film form is to provide an opportunity to test whether such a belief still holds at low dimension where very large surface to bulk ratio can be well controlled. These films have superconducting properties similar to the bulk materials. The onset $T_{\mathrm{c}}$, are about $3.8 \mathrm{~K}$ and $3.6 \mathrm{~K}$ and the upper critical field $H_{\mathrm{c} 2}$ at $T=0$ $\mathrm{K}$ is estimated to be $\sim 1.65 \mathrm{~T}$ and $1.50 \mathrm{~T}$ for the (100) and (111) orientations respectively. These thin films provide an ideal platform for study of TCIs and topological superconductivity.

We used pulsed laser deposition to grow the SIT thin films. A KrF excimer laser (wavelength: $248 \mathrm{~nm}$ ) with an energy density of $\sim 3.0 \mathrm{~J} / \mathrm{cm}^{2}$ was used for thin film deposition. The 
substrate temperature was varied from $200{ }^{\circ} \mathrm{C}$ to $350{ }^{\circ} \mathrm{C}$. The base pressure of the deposition chamber was $\sim 5 \times 10^{-9}$ Torr and $\sim 5 \times 10^{-8}$ Torr at the elevated temperature during the deposition. The substrate was heated by another laser beam, which was shut off immediately after the deposition. The deposition rate was about $0.1 \mathrm{~nm} /$ pulse. Typical film thickness is about $170 \mathrm{~nm}$. Structural characterization was performed using X-ray diffractometry. The films were patterned by laser cutting. The bridges were made to be about $300 \mu \mathrm{m}$ long and $100 \mu \mathrm{m}$ wide for transport characterization. Resistivity was measured by the standard four-probe method in a physical property measurement system (PPMS, Quantum Design). Magnetization was measured in a superconducting quantum interference device (MPMS, Quantum Design).

Figure 1 shows $\theta-2 \theta$ x-ray diffraction scans for the SIT thin films grown on the (100) and (111) $\mathrm{BaF}_{2}$ substrates. In order to fully confirm the alignment in the (111) orientation, we did the $\theta-2 \theta$ scan from $20^{\circ}$ to $130^{\circ}$ to include the (444) peaks from the film and the substrate. Only peaks along the substrate orientations from the thin films and substrates are present, indicating that the alignments for films of both orientations are good. Lattice constants of the SIT thin films, assuming a cubic system, are estimated to be $\sim 0.626 \mathrm{~nm}$ and $0.628 \mathrm{~nm}$ for the films of (100) and (111) orientations respectively, generally consistent with that of the bulk materials [16]. Fig. 2(a) shows the rocking curves of (200) peaks from the (100) oriented SIT film and $\mathrm{BaF}_{2}$ substrate, whereby Fig. 2(b) shows the rocking curves of (222) peaks from the (111) oriented SIT film and $\mathrm{BaF}_{2}$ substrate. The full width at half maximums (FWHM) are approximately $0.04^{\circ}$ and $0.03^{\circ}$ for the (100) and (111) $\mathrm{BaF}_{2}$ crystalline substrates, and $\sim 0.62^{\circ}$ and $0.27^{\circ}$ for the (100) and (111) oriented films. The (111) oriented films have a better crystallinity than the (100) oriented films. We noticed two very small peaks from an unknown phase in the (100) oriented film as indicated by the asterisks in Fig. 1a (these do not appear in the (111) oriented films). We suspect a $\mathrm{TeO}_{2}$ impurity. SnTe is 
known to have many Sn vacancies. There could be excess Te during the deposition, leading to the $\mathrm{TeO}_{2}$ formation. As noted, (111) oriented films have a better crystallinity, therefore more likely have less Sn vacancies, hence less excess Te. Further studies are needed for clarification.

Figure 3(a) shows the temperature dependence of the resistivity for the SIT thin films in both (100) and (111) orientations, while Fig. 3(b) shows the detail of the superconducting transition. Similar temperature dependences were observed for both orientations over the entire temperature regime, which are also similar to that of the bulk [16]. The residual resistivities above the superconducting transition are about $500 \mu \Omega \mathrm{cm}$ for (100) and $430 \mu \Omega \mathrm{cm}$ for (111) orientations. These are about double that of the bulk reported in Ref. [16], while lower than the result from the single crystal in Ref. [18]. The onset of superconducting transition $T_{\mathrm{c}}$ 's are $3.8 \mathrm{~K}$ and $3.6 \mathrm{~K}$ for the (100) and (111) oriented films, respectively, slightly lower than that of bulk $T_{\mathrm{c}}, \sim 4.1 \mathrm{~K}$ (the target used in our thin film deposition). The zero resistance $T_{\mathrm{c}}$ are around $3.1 \mathrm{~K}$ and $2.6 \mathrm{~K}$ with the transition widths about $0.7 \mathrm{~K}$ and $1 \mathrm{~K}$ for the (100) and (111) orientations respectively, larger than that of the single crystals as is common for superconducting thin films. Further optimization of growth condition for these films will likely to improve their quality and $T_{\mathrm{c}}$. Fig. 3(c) shows the magnetic superconducting transition of the same two films. The clearly observed diamagnetism signals a global superconducting transition at $\sim 2.8 \mathrm{~K}$ for the (100) and $2.4 \mathrm{~K}$ for the (111) oriented films. Both $T_{\mathrm{c}}$ 's from the magnetic measurement are a little lower than those from the resistivity measurements, also a common observation in superconducting thin films with thickness comparable to the London penetrations depth. It is interesting to note that the $T_{\mathrm{c}}$ of the (111) oriented SIT film is lower than that of the (100) oriented film although it has a better crystallinity and fewer impurities, based on the structural analysis. It is possible that the indium doping level is lower in the (111) oriented films than that in the (100) oriented films. To confirm, this will need ultra-high resolution 
chemical analysis to accurately detect the indium content.

Figure 4 shows the magneto-resistive transitions at various temperatures from $1.8 \mathrm{~K}$ to $4 \mathrm{~K}$ for the SIT thin films in both (100) and (111) orientations. The magnetic fields are applied perpendicular to the substrate (film) surfaces for both (100) and (111) films. The superconducting transition shows the conventional behavior for a type II superconductor: the magnetic field reduces the $T_{\mathrm{c}}$ linearly but does not significantly change the superconducting transition width. The upper critical field $H_{\mathrm{c} 2}$ and the irreversibility field $H_{\mathrm{irr}}$ were determined from such measurements. Specifically, $H_{\mathrm{c} 2}(T)$ is defined as the onset of the resistive transition at each fixed temperature. In this case the onset $T_{\mathrm{c}}$ is defined as the temperature at which the resistivity drops to the $90 \%$ of the normal state level. The $H_{\text {irr }}(T)$ is defined as the zero resistance $T_{\mathrm{c}}$, down to the resolution of our measurement, $\sim 50 \mathrm{nV} / \mathrm{cm}$. The results are shown in Fig. 5. It is notable that the $H_{\mathrm{c} 2}$ is slightly higher in the (100) oriented film than in the single crystals at low temperatures, although our thin films have a slightly lower $T_{\mathrm{c}}$. It is possible that the thin films have a higher level of defect or disorder that results in a shorter mean free path, leading to a lower effective superconducting coherence length $\xi$, as $H_{\mathrm{c} 2} \sim 1 / \xi^{2}$. It is noted that $H_{\mathrm{c} 2}(T)$ are almost linear in both (100) and (111) oriented films in our measured temperature range below $T_{\mathrm{c}}$, that appears to suggest some unconventional behavior. Preliminary measurements over a broad phase space of temperature and magnetic field seem to show complicated transport behaviors of these films that have not been observed in the bulk samples. Detailed studies with careful analysis are needed to reach any conclusion. The upper critical field at zero temperature $H_{\mathrm{c} 2}(T=0)$ is estimated using the Werthamer-Helfand-Hohenberg approximation, $H_{\mathrm{c} 2}(0)=-0.69 T_{\mathrm{c}} d H_{\mathrm{c} 2} / d T \mid T=T_{\mathrm{c}}$ [26], although this approximation may not be strictly applicable for superconductors with linear temperature dependence of $H_{\mathrm{c} 2}(\mathrm{~T})$ over a abroad temperature range. From the measured curves for $H_{\mathrm{c} 2}(\mathrm{~T})$, we 
obtain an initial slope (at $T \approx T_{\mathrm{c}}$ ) of $-0.63 \mathrm{~T} / \mathrm{K}$ and $-0.61 \mathrm{~T} / \mathrm{K}$ for (100) and (111) oriented films. Using $T_{\mathrm{c}}=3.8 \mathrm{~K}$ and $3.6 \mathrm{~K}$, the upper critical field $H_{\mathrm{c} 2}(0)$ is estimated to be approximately $1.65 \mathrm{~T}$ and $1.50 \mathrm{~T}$ for the (100) and (111) oriented films respectively, as high or higher than that of the single crystal, $\sim 1.49 \mathrm{~T}[16,18]$. The $H_{\text {irr }}(\mathrm{T})$ has an upward curvature compared with the almost linear curvature of the $H_{\mathrm{c} 2}(\mathrm{~T})$ for films of both orientations. This is in contrast to the linear behaviors observed in Ref. [18] for both $H_{\mathrm{irr}}(\mathrm{T})$ and $H_{\mathrm{c} 2}(\mathrm{~T})$. This probably reflects a different flux pinning mechanism in the films versus in the single crystals, and more studies are suggested.

Transport properties of thin films can also be influenced by the interface between the thin film and substrate. Preliminary transmission electron microscopy examination of our thin films found a thin amorphous region between the SIT film and the substrate. This amorphous region is about a few nm thick and mostly composed of Sn, Te and Ba, indicating some kind of reaction between the film and the $\mathrm{BaF}_{2}$ substrate. Elemental $\mathrm{Sn}$ is superconducting with much smaller $H_{\mathrm{c} 2}$. Sn-In alloy has a higher $H_{\mathrm{c} 2}$, however, we do not observe significant amount of In in the amorphous region. This amorphous region unlikely plays an important role in the observed transport properties, but clearly needs further study.

In conclusion, we report on the superconducting properties of epitaxial thin films of $\mathrm{Sn}_{0.55} \mathrm{In}_{0.45} \mathrm{Te}$ grown on the (100) and (111) oriented $\mathrm{BaF}_{2}$ substrates by pulsed laser deposition. Thin films have properties similar to those of the bulk materials. The onset superconducting transition temperature is about $3.8 \mathrm{~K}$ and $3.6 \mathrm{~K}$ for the (100) and (111) oriented films respectively. Magneto-resistive measurement shows that the thin films may have an upper critical field higher than that of the bulk. Since thin films have a much larger surface-to-bulk ratio, we believe that indium-doped SnTe films can provide a rich platform for studies of the TCIs and topological superconductivity, as well as for potential device applications, such as superconducting proximity 
and quantum computing.

This work was supported by the U.S. Department of Energy, Office of Basic Energy Science, Division of Materials Science and Engineering, under Contract No. DE-SC0012704. The authors would like to thank Dr. Arnold Moodenbaugh for critical reading of this manuscript.

\section{References}

[1] M. Z. Hasan and C. L. Kane, Rev. Mod. Phys. 82, 3045 (2010).

[2] J. E. Moore, Nature (London) 464, 194 (2010).

[3] Di Xiao, Wenguang Zhu, Ying Ran, Naoto Nagaosa and Satoshi Okamoto, Nat. Commun. 2, 596 (2011).

[4] S.-Y. Xu, C. Liu, N. Alidoust, M. Neupane, D. Qian, I. Belopolski, J. D. Denlinger, Y. J. Wang, H. Lin, L. A. Wray, G. Landolt, B. Slomski, J. H. Dil, A. Marcinkova, E. Morosan, Q. Gibson, R. Sankar, F. C. Chou, R. J. Cava, A. Bansil, and M. Z. Hasan, Nat. Commun. 3, 1192 (2012).

[5] X.-L. Qi and S.-C. Zhang, Rev. Mod. Phys. 83, 1057 (2011).

[6] L. Fu and C. L. Kane, Phys. Rev. Lett. 100, 096407 (2008).

[7] L. Fu and E. Berg, Phys. Rev. Lett. 105, 097001 (2010).

[8] T. H. Hsieh and L. Fu, Phys. Rev. Lett. 108, 107005 (2012).

[9] S. Sasaki, Z. Ren, A. A. Taskin, K. Segawa, L. Fu, and Y. Ando, Phys. Rev. Lett. 109, 217004 (2012).

[10] L. Fu, Phys. Rev. Lett. 106, 106802 (2011).

[11] T. H. Hsieh, H. Lin, J. Liu, W. Duan, A. Bansil, and L. Fu, Nat. Commun. 3, 982 (2012).

[12] Y. Tanaka, Z. Ren, T. Sato, K. Nakayama, S. Souma, T. Takahashi, K. Segawa, and Y. Ando, Nat. Phys. 8, 800 (2012). 
[13] T. Sato,Y. Tanaka, K. Nakayama, S. Souma, T. Takahashi, S. Sasaki, Z. Ren, A. A. Taskin, K. Segawa, and Y. Ando, Phys. Rev. Lett. 110, 206804 (2013).

[14] A. S. Erickson, J.-H. Chu, M. F. Toney, T. H. Geballe, and I. R. Fisher, Phys. Rev. B 79, 024520 (2009).

[15] G. Balakrishnan, L. Bawden, S. Cavendish and M. R. Lees, Phys. Rev. B 87, 140507 (2013).

[16] R. D. Zhong, J. A. Schneeloch, X. Y. Shi, Z. J. Xu, C. Zhang, J. M. Tranquada, Q. Li and G. D. Gu, Phys. Rev. B 88, 020505 (2013).

[17] Mario Novak, Satoshi Sasaki, Markus Kriener, Kouji Segawa, and Yoichi Ando, Phys. Rev. B 88, 140502 (2013).

[18] V. K. Maurya, Shruti, P. Srivastava and S. Patnaik, EPL 108, 37010 (2014).

[19] R. D. Zhong, J. A. Schneeloch, T. S. Liu, F. E. Camino, J. M. Tranquada and G. D. Gu, Phys. Rev. B 90, 020505 (2014).

[20] Jie Shena and Judy J. Cha, Nanoscale 6, 14133 (2014).

[21] Junwei Liu, Timothy H. Hsieh, PengWei,Wenhui Duan, Jagadeesh Moodera, and Liang Fu, Nat. Matter. 13, 178 (2014).

[22] Chen Fang, Matthew J. Gilbert, and B Andrei Bernevig, Phys. Rev. Lett. 112, 046801 (2014). [23] Hua Guo, Chen-Hui Yan, Jun-Wei Liu, Zhen-Yu Wang, Rui Wu, Zhi-Dong Zhang, Li-Li Wang, Ke He, Xu-Cun Ma, Shuai-Hua Ji, Wen-Hui Duan, Xi Chen, and Qi-Kun Xue, APL Mater. 2, 056106 (2014).

[24] A. A. Taskin, F. Yang, S. Sasaki, K. Segawa and Y. Ando, Phys. Rev. B 89, 121302 (2014).

[25] B. A. Assaf, F. Katmis, P. Wei, B. Satpati, Z. Zhang, S. P. Bennett, V. G. Harris, J. S. Moodera, and D. Heiman, Appl. Phys. Lett. 105, 102108 (2014).

[26] N. R. Werthamer, E. Helfand, and P. C. Hohenberg, Phys. Rev. 147, 295 (1966). 

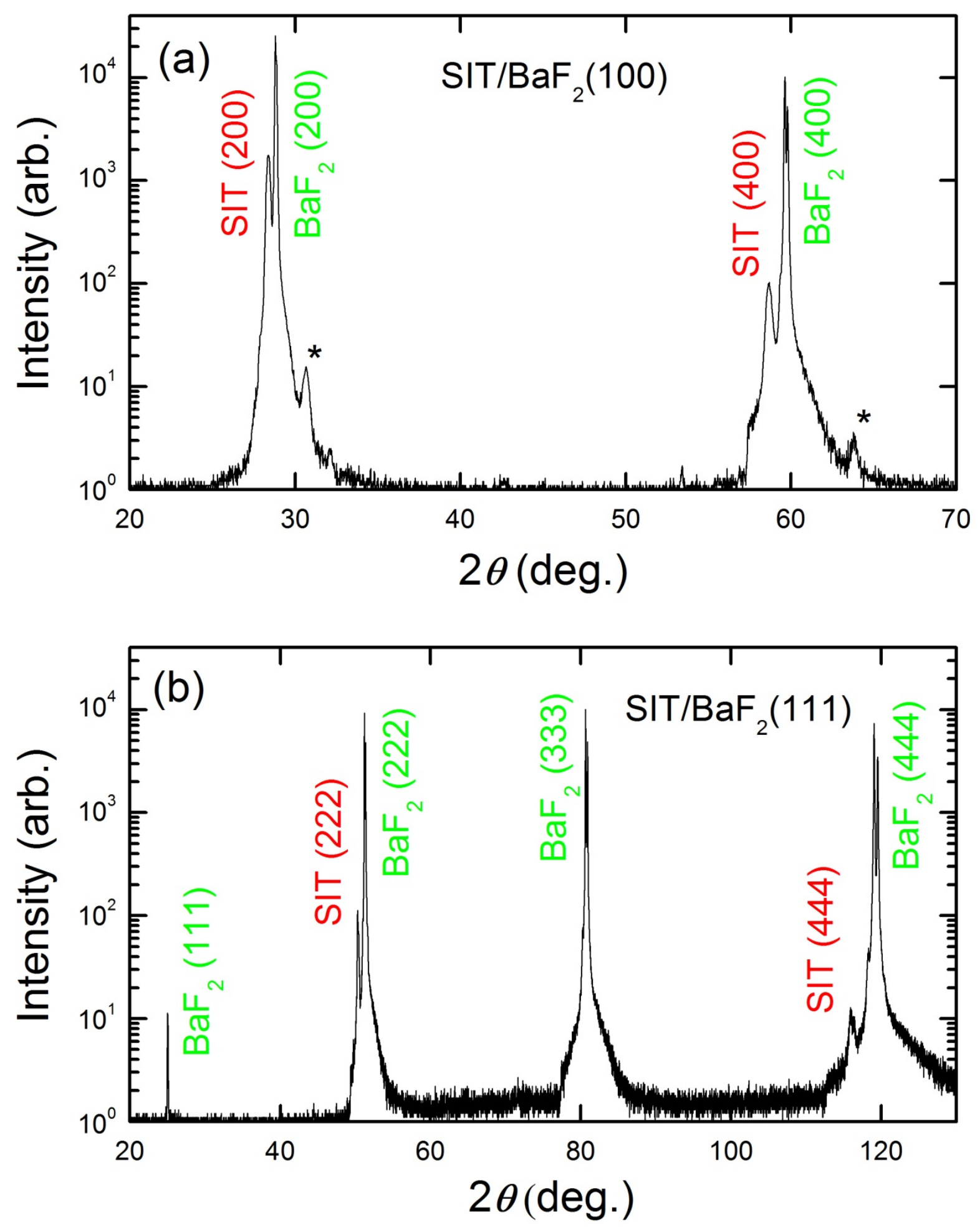

FIG. $1 \mathrm{X}$-ray $\theta-2 \theta$ scan for $\mathrm{Sn}_{0.55} \mathrm{In}_{0.45} \mathrm{Te}$ thin films grown on the (a) (100) and (b) (111) $\mathrm{BaF}_{2}$ substrates. 

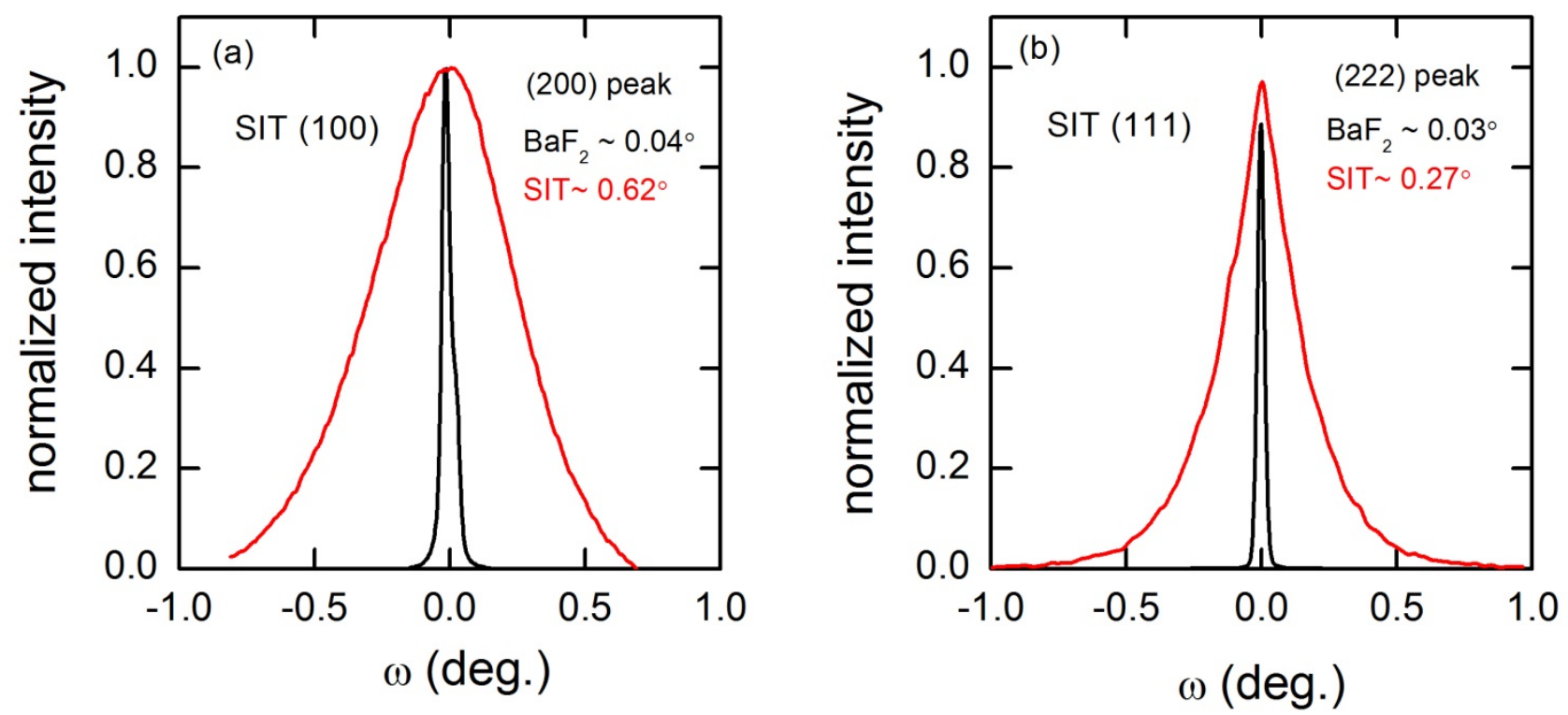

FIG. 2 (a) Rocking curve for the (200) peak of a $\mathrm{Sn}_{0.55} \mathrm{In}_{0.45} \mathrm{Te}$ film grown on a (100) $\mathrm{BaF}_{2}$ substrate. (b) Rocking curve for the (222) peak of a $\mathrm{Sn}_{0.55} \mathrm{In}_{0.45}$ Te film grown on a (111) $\mathrm{BaF}_{2}$ substrate. 

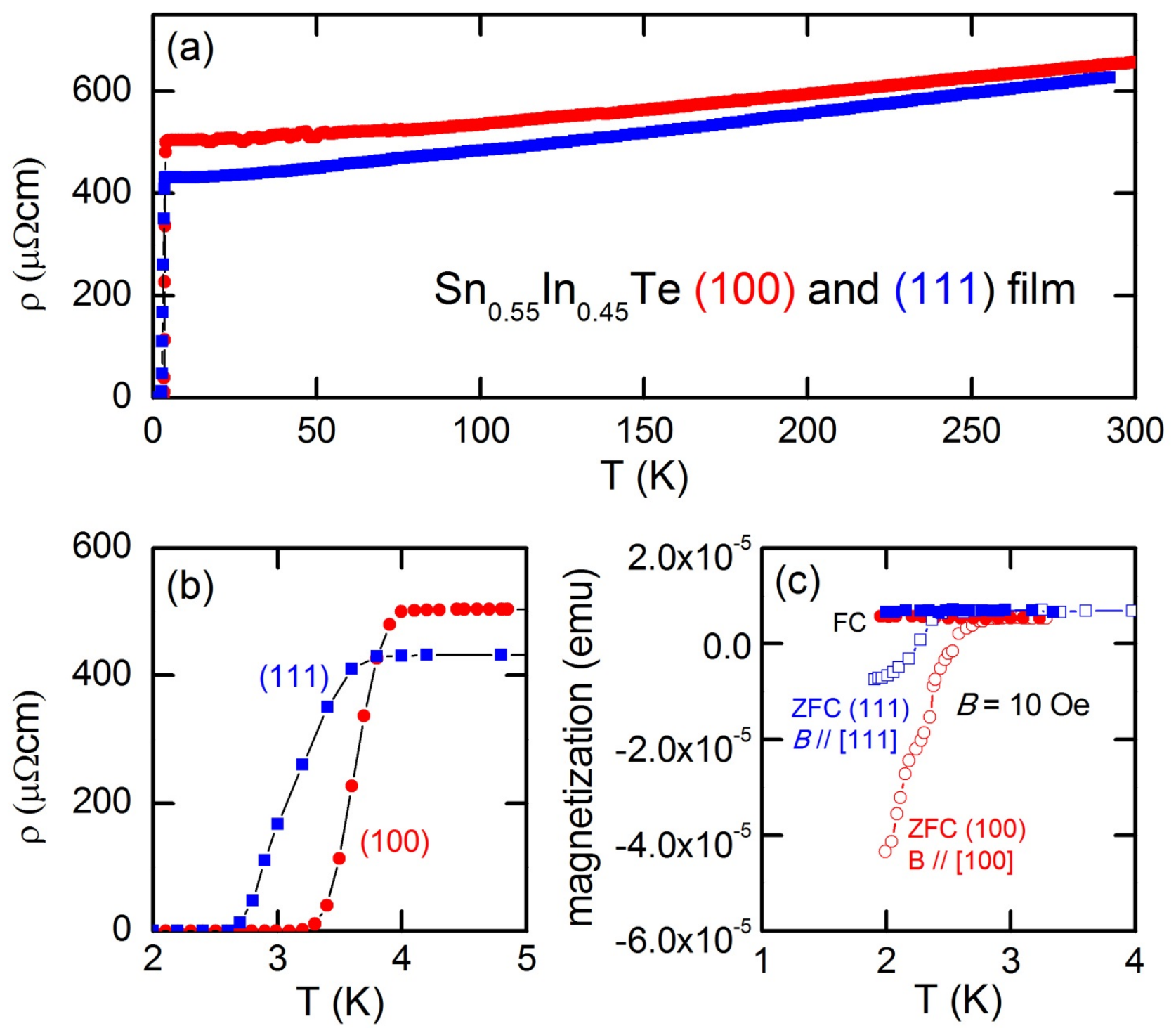

FIG. 3 (a) Temperature dependence of the resistivity for the (100) and (111) $\mathrm{Sn}_{0.55} \mathrm{In}_{0.45} \mathrm{Te}$ thin films grown on the $\mathrm{BaF}_{2}$ substrates. (b) A detail of the superconducting transition. $T_{\mathrm{c}}$ onsets are about 3.8 $\mathrm{K}$ and $3.6 \mathrm{~K}$ and zero resistance $T_{\mathrm{c}}$ are about $3.1 \mathrm{~K}$ and $2.6 \mathrm{~K}$ for the (100) and (111) oriented films respectively. (c) The magnetic transitions in both field cooling (FC) and zero field cooling (ZFC) for the same two films. 


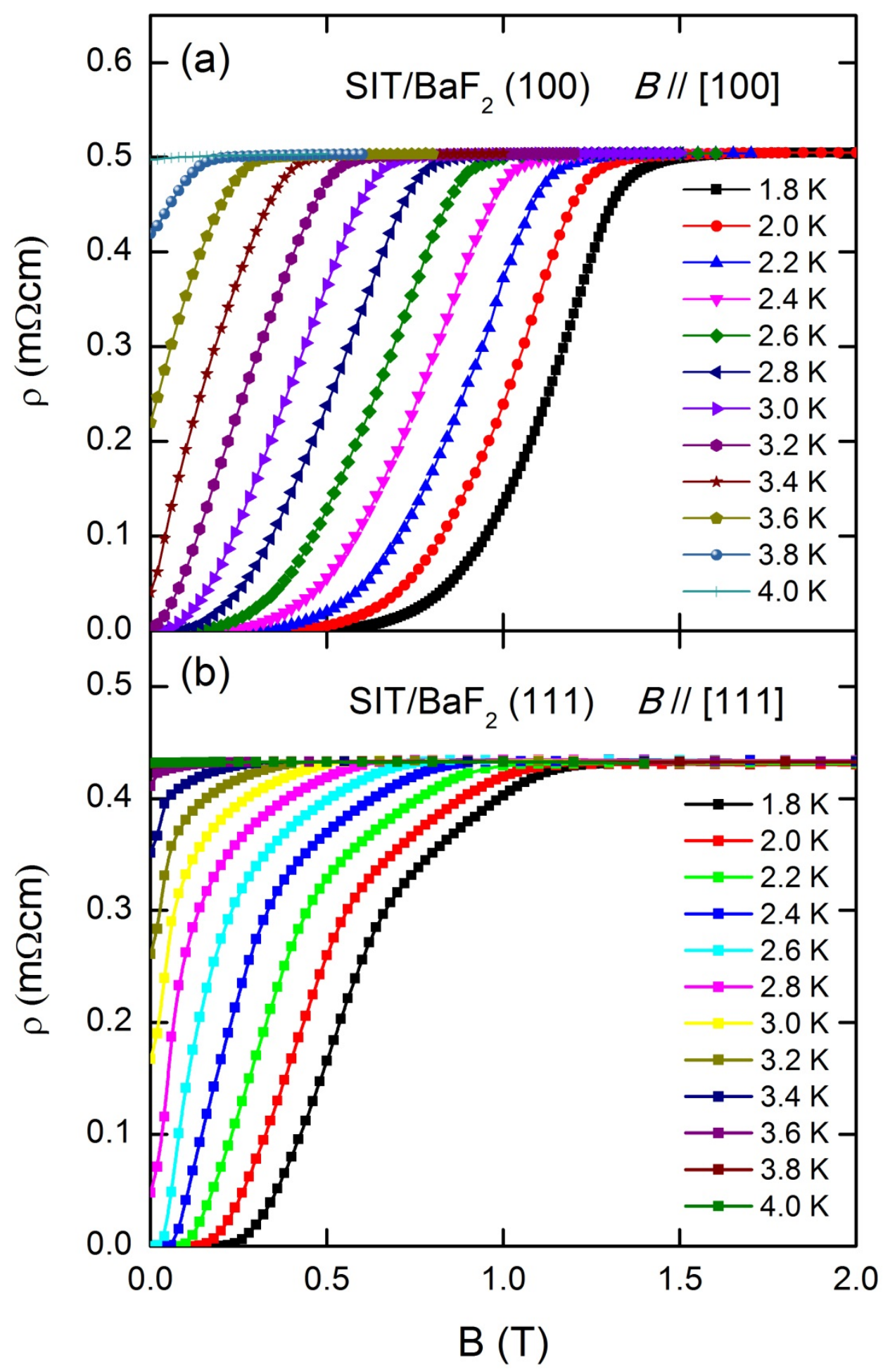

FIG. 4 Resistive transitions of the superconducting (100) and (111) $\mathrm{Sn}_{0.55} \mathrm{In}_{0.45} \mathrm{Te}$ thin films grown on the $\mathrm{BaF}_{2}$ substrates as a function of applied magnetic field $B$ at different temperatures. 


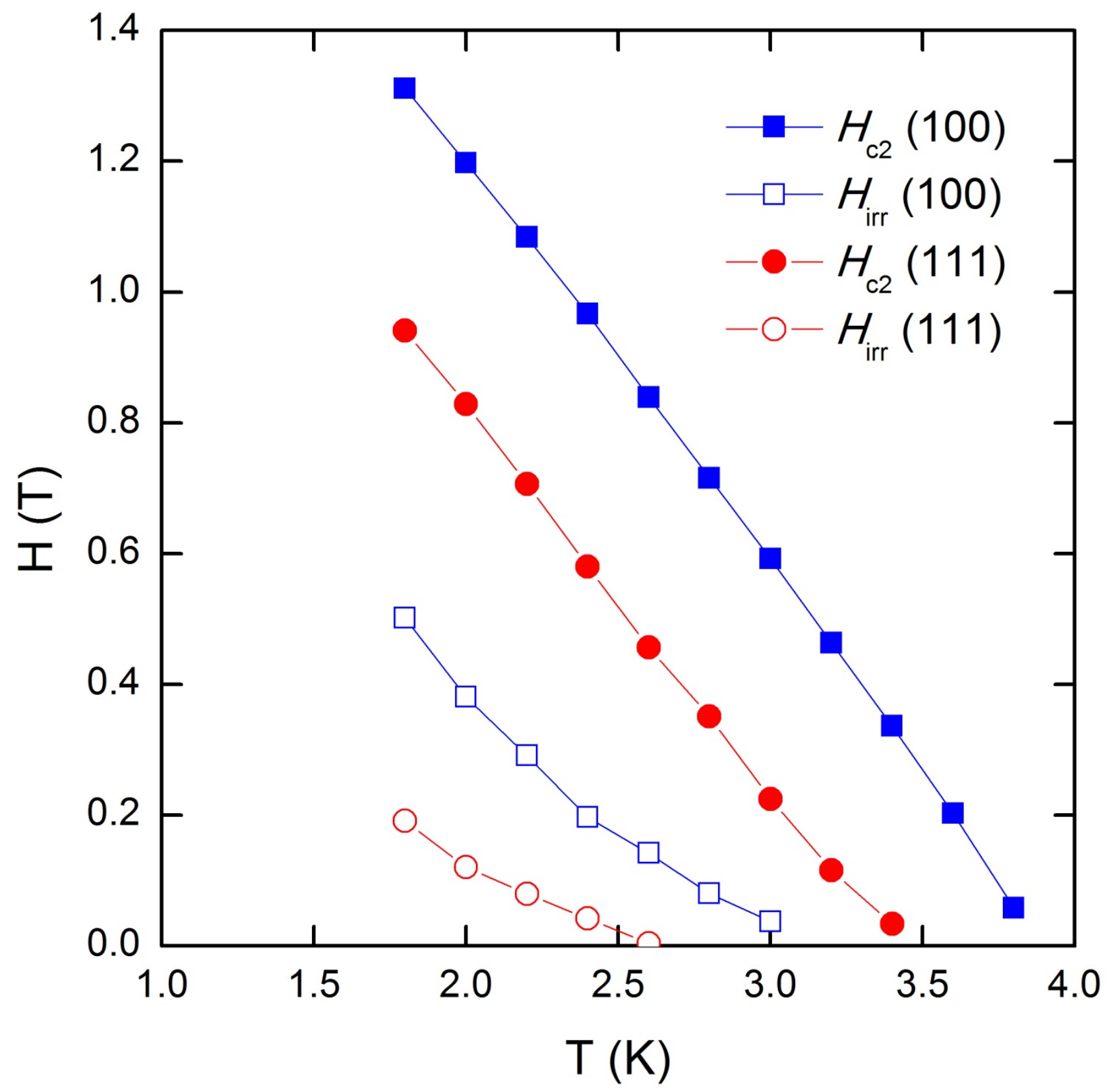

FIG. 5 The upper critical field $H_{\mathrm{c} 2}$ and the irreversibility field $H_{\text {irr }}$ of the (100) and (111) $\mathrm{Sn}_{0.55} \mathrm{In}_{0.45} \mathrm{Te}$ thin films grown on the $\mathrm{BaF}_{2}$ substrates as a function of temperature. 